\title{
Inverse problems of plane elasticity for the determination of contact stresses
}

\author{
A. N. Galybin \\ Wessex Institute of Technology, Southampton, UK
}

\begin{abstract}
This article presents some results of numerical experiments on the reconstruction of contact stresses by using synthetic data on displacement monitoring on a traction-free surface. The corresponding boundary value problem is ill-posed therefore a regularisation procedure based on the SVD decomposition is employed. Statistical analysis of results has been performed.

Keywords: contact problem, plane elasticity, boundary value problems, inverse problems, SVD regularisation.
\end{abstract}

\section{Introduction}

There are many applications where data on stresses or displacements may not be available on the entire boundary of a body (including internal boundaries). Such problems appear in strain-stress measurements, interferometry, rock mechanics, monitoring the fracture development in strength tests etc. They require the consideration of a specific boundary value problem, BVP, which is overspecified on a part of the boundary and underspecified on the rest of it.

The theory of classical BVPs of plane elastostatics is fully presented in the classical monograph by Muskhelishvili [1], it assumes that two scalar boundary conditions are given on the entire boundary of a domain. In contact mechanics the BVPs are usually formulated as the mixed type problems when displacements are given in the contact zone and tractions on the rest of the surface. Other formulations of contact conditions are discussed in detail in Johnson [2]. In all these cases the boundary value problem is well posed, therefore it possesses a unique and stable solution.

Despite classical boundary conditions describe a wide class of mechanical phenomena there is still the necessity to use additional assumptions in 
formulation of the boundary conditions. This can lead to paradoxical results, as for instance, in the problem for a flat stamp indented into the boundary of a halfplane without sliding. The exact solution shows oscillation of the contact stresses in a small zone near the stamp edges, which does not obey the assumption that the stamp and the half-plane are in contact everywhere. This effect should not be overlooked if one intends to investigate the stress distribution under the stamp edges, which presents the case of interest, for instance, for fracture development. Thus, corrections of the boundary conditions may be required to provide the consistence of obtained results. However this presumes that some new assumptions will be needed for describing the contact conditions.

Alternatively the BVPs of this type can be solved with the use of the displacement monitoring data over the free boundary outside the contact zone. In this case both the displacements and the contact stresses remain unknown under the stamp and the BVP is underdetermined on a part of the boundary and overdetermined on the rest of it. Perhaps, the first comprehensive analysis of solvability of these problems has been reported by Shvab [3] for an isotropic elastic domain with the following boundary conditions: displacement vector is given on a part of the boundary simultaneously with the stress vector; the rest of the boundary has no conditions posed. This problem can be viewed as consecutive problems for holomorphic vectors, on which the proof of uniqueness can also be based, e.g. [4]. Methods involving complex variables for investigation of this problem in $2 \mathrm{D}$ have also been applied [5,6]. The problem can be referred to as conditionally ill-posed [3], one can rarely find analytical solutions for it (with exceptions for simple domains, e.g. for wedge-like domains [5]), therefore the development of stable numerical methods has been the main focus during the last years. The considerable progress has recently been achieved by researches from University of Leeds (UK) in the development of regularisation techniques, iterative methods and algorithms for solving nonclassical BVPs of this type, see for instance [7-10]. In particular, it has been shown that methods based on the Tikhonov regularisation provide stable solutions in elastostatics, [8,9]. Other studies, e.g. [10-12], confirm this conclusion, in particular, it has been found $[10,12]$ that the use of the SVD regularisation presents a valuable computational tool in elastostatics. In this study the SVD regularisation is applied for stress identification in contact mechanics problems.

\section{Mathematical formulation}

\subsection{Inverse BVP: displacements are known on traction-free surfaces}

Let $\Gamma$ be a closed contour separating the complex plane into interior $\Omega^{+}$and exterior $\Omega^{-}$domains. In contact problems one of these domains can be associated with one contacting (plane) body, say, $\Omega^{+}$if one considers finite bodies or $\Omega^{-}$if infinite. We further consider the stress state of only one of the contacting bodies. The action of another body is replaced by unknown stresses distributed over the contact zone, therefore the shape of this body is unimportant in the present 
formulation and without loss of generality it is assumed that it occupies the complement of $\Omega^{+}$(or $\Omega^{-}$) with respect to the whole plane. For the same reason it is assumed that elastic moduli of contacting bodies are the same. Stress states in both domains can be expressed through sectionally holomorphic functions (complex potentials) $\varphi(\mathrm{z})$ and $\psi(\mathrm{z})$ of complex variable $\mathrm{z}=x+i y$ by the KolosovMuskhelishvili solution [1]. In particular on the boundary one has the following expressions for tractions, $\mathbf{t}=\left(t_{1}, t_{2}\right)$, and displacements, $\mathbf{u}=\left(u_{1}, u_{2}\right)$

$$
F(\zeta)=\varphi(\zeta)+\overline{\mathrm{T}(\zeta)}, \quad W(\zeta)=\kappa \varphi(\zeta)-\overline{\mathrm{T}(\zeta)}
$$

Hereafter $F=t_{1}+i t_{2} ; W=2 G\left(u_{1}+i u_{2}\right) ; \zeta \Subset \Gamma$ is a point on the boundary; $G$ is shear modulus, elastic constant $\kappa=3-4 v$ to for plane strain and $\kappa=(3-v)(1+v)^{-1}$ for plane stress, $v$ is Poisson's ratio; $\mathrm{T}(\zeta)$ is boundary value of bi-holomorphic function

$$
\mathrm{T}(z, \bar{z})=\bar{z} \varphi^{\prime}(z)+\psi(z)
$$

From (1) it is evident that

$$
(1+\kappa) \varphi(\zeta)=F(\zeta)+W(\zeta), \quad(1+\kappa) \overline{\mathrm{T}(\zeta)}=\kappa F(\zeta)-W(\zeta), \quad(1+\kappa) \mathrm{T}(\zeta)=\kappa \overline{F(\zeta)}-\overline{W(\zeta)}
$$

If tractions and displacements on the entire boundary are know it is easy to determine complex potentials everywhere inside the domain considered by using the integral Cauchy formula as follows

$$
\begin{aligned}
& \varphi(z)= \pm \frac{1}{2(1+\kappa) \pi i} \int_{\Gamma} \frac{F(t)+W(t)}{t-z} d t, \quad \varphi(\infty)=0 \\
& \mathrm{~T}(z, \bar{z})= \pm \frac{1}{2(1+\kappa) \pi i} \int_{\Gamma} \frac{\kappa \overline{F(t)}-\overline{W(t)}-(\bar{t}-\bar{z})\left(F^{\prime}(t)+W^{\prime}(t)\right)}{t-z} d t, \quad T(\infty)=0
\end{aligned}
$$

Hereafter upper/lower sings refer to domains $\Omega^{ \pm}$respectively; $\Gamma$ is traversed in counterclockwise direction.

It should be noted that the functions $F(t)$ and $W(t)$ are dependent on the entire boundary, however they can be chosen independently on a part of the boundary, which leads to the boundary value problem depicted in Figure 1.

Let us represent this problem as follows

$$
F(\zeta)=\left\{\begin{array}{cc}
0 & \zeta \in \Gamma_{1} \\
X(\zeta) & \zeta \in \Gamma_{2}
\end{array}, \quad W(\zeta)=\left\{\begin{array}{cc}
w(\zeta) & \zeta \in \Gamma_{1} \\
Y(\zeta) & \zeta \in \Gamma_{2}
\end{array}\right.\right.
$$

where $X(\zeta)$ and $Y(\zeta)$ are unknown functions; it is also assumed that displacements are monitored on a part of the traction-free surface and therefore $w(\zeta)$ is known there. The existence of traction-free surfaces does not narrow the formulation because due to linearity one can superimpose a solution for known tractions on the solution of the problem (5).

The problem can be reduced to the consequent determination of holomorphic functions by analytic continuation. It is shown, e.g. [3-5], that this problem has a 
unique solution. Here we derive an integral equation for the determination of unknown functions $X(\zeta)$ and $Y(\zeta)$ on the basis of representations (4).

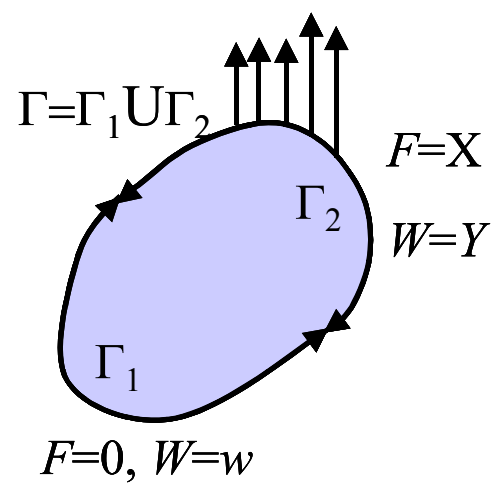

Figure 1: The problem.

\subsection{Integral equations}

Let us introduce the following integral operators

$$
\begin{gathered}
\mathbf{S}^{k} g=\frac{1}{\pi i} \int_{\Gamma_{k}} \frac{g(t)}{t-\zeta} d t, \quad k=1,2 ; \quad \mathbf{S} g=\mathbf{S}^{1} g+\mathbf{S}^{2} g, \quad \mathbf{S}(\mathbf{S} g)=g \\
\mathbf{R}_{1}^{k} g=\frac{1}{\pi i} \int_{\Gamma_{k}} \frac{\bar{t}-\bar{\zeta}}{t-\zeta} g^{\prime}(t) d t=\frac{1}{\pi i} \int_{\Gamma_{k}}\left(\frac{d \bar{t}}{d t}-\frac{\bar{t}-\bar{\zeta}}{t-\zeta}\right) \frac{g(t)}{t-\zeta} d t \\
\mathbf{R}_{2}^{k} g=\frac{1}{\pi i} \int_{\Gamma_{k}}\left(\frac{t-\zeta}{\bar{t}-\bar{\zeta}} \frac{d \bar{t}}{d t}-1\right) \frac{g(t)}{t-\zeta} d t, \quad \mathbf{R}_{j} g=\mathbf{R}_{j}^{1} g+\mathbf{R}_{j}^{2} g, \quad k, j=1,2
\end{gathered}
$$

where $\mathbf{S g}$ is singular and $\mathbf{R}_{\mathbf{j}} \mathbf{g}$ are regular operators. The latter are used in the representations for bi-holomorphic function and complex conjugation of $\mathbf{S g}$

$$
\begin{gathered}
\mathbf{S T}=\bar{\zeta} \mathbf{S} \varphi^{\prime}+\mathbf{S} \psi+\mathbf{R}_{1} \varphi^{\prime} \\
\overline{\mathbf{S} g}=-\mathbf{S} \bar{g}-\mathbf{R}_{2} \bar{g}
\end{gathered}
$$

With the use of these operators one can present the boundary values of the functions in (3) in the following form

$$
\varphi= \pm \mathbf{S} \varphi, \quad \mathrm{T}= \pm \mathbf{S T} \mp \mathbf{R}_{1} \varphi, \quad \overline{\mathrm{T}}= \pm \overline{\mathbf{S T}} \mp \overline{\mathbf{R}_{1} \varphi}=\mp \mathbf{S} \overline{\mathrm{T}} \mp \overline{\mathbf{R}_{1} \varphi} \mp \mathbf{R}_{2} \overline{\mathrm{T}}
$$

Substitution of (3) into (10) results in 


$$
\begin{aligned}
& \mathbf{S}(F+W)= \pm(F+W) \\
& \left(\mathbf{S}+\mathbf{R}_{2}\right)(\kappa F \mp W)+\overline{\mathbf{R}_{1}(F+W)}=\mp(\kappa F+W)
\end{aligned}
$$

Now one can derive a system of integral equations on the traction-free boundary $\Gamma_{2}$ by decomposition of the left hand sides in (11) and applying boundary conditions (5) on different parts of the entire boundary.

$$
\left\{\begin{array}{l}
\mathbf{S}^{2}(X+Y)= \pm w-\mathbf{S}^{1} w \\
\left(\mathbf{S}^{2}+\mathbf{R}_{2}^{2}\right)(\kappa X-Y)+\overline{\mathbf{R}_{1}^{2}(X+Y)}= \pm w+\left(\mathbf{S}^{1}+\mathbf{R}_{2}^{1}\right) w-\overline{\mathbf{R}_{1}^{1} w}
\end{array}\right.
$$

It should be noted that operators $\mathbf{S}^{2}$ are not singular on $\Gamma_{2}$, with possible exception of the ends of the contour (fixed singularities), therefore the system (12) is a system of integral equations of the Fredholm type. Both equations in (12) are conditionally ill-posed because they are of the first kind [13], therefore there exists a unique solution of this system but it is unstable, which requires regularisation. One can formally introduce two stable operators $\mathbf{P}$ and $\mathbf{Q}$ that represent regularised inverse operators of $\mathbf{S}^{2}$ and $\mathbf{S}^{2}+\mathbf{R}_{2}{ }^{2}$ respectively, i.e.

$$
\mathbf{S}^{2} g \approx \mathbf{P}^{-1} g, \quad\left(\mathbf{S}^{2}+\mathbf{R}_{2}^{2}\right) g \approx \mathbf{Q}^{-1} g
$$

By applying these operators to equations in (12) one obtains an approximate solution of the system in the following form

$$
\left(\begin{array}{l}
X \\
Y
\end{array}\right)=\frac{1}{1+\kappa}\left(\begin{array}{cc}
\mathbf{P} & \mathbf{Q} \\
\kappa \mathbf{P} & -\mathbf{Q}
\end{array}\right)\left(\begin{array}{c}
\frac{f_{1}}{f_{2}-\mathbf{R}_{1}^{2}\left(\mathbf{P} f_{1}\right)}
\end{array}\right)
$$

where the right hand sides in equations (12) are denoted as

$$
f_{1}= \pm w-\mathbf{S}^{1} w, \quad f_{2}= \pm w+\left(\mathbf{S}^{1}+\mathbf{R}_{2}^{1}\right) w-\overline{\mathbf{R}_{1}^{1} w}
$$

As soon as tractions and displacements on $\Gamma_{1}$ are found one can determine complex potentials by formulae (3)-(5). It should be emphasised that solution (14) is stable with respect to small perturbations in monitored displacements $w$ that appear due to experimental errors or limited resolution in measurements. Construction of the inverse operators $\mathbf{P}$ and $\mathbf{Q}$ is, obviously, an important part in engineering applications. The next section presents an example employing the SVD regularisation, which, as shown in $[10,12]$, is an effective tool for this type of problems.

\section{Numerical analysis}

\subsection{Integral equation for half-plane}

Let us consider the lower half-plane under a symmetric load distributed on $(-1,1)$ while displacements are monitored on $(1,1+L)$ as shown in Figure 2. In this case 
the boundaries are $\Gamma_{1}=(1,1+L)$ and $\Gamma_{2}=(-\infty, 1) \cup(1+L, \infty)$; it is also evident that the regular operators $\mathbf{R}_{1}=\mathbf{R}_{2}=0$. Hence the system (12) assume the following form

$$
\left\{\begin{array}{l}
\mathbf{S}^{2}(X+Y)=-w-\mathbf{S}^{1} w \\
\mathbf{S}^{2}(\kappa X-Y)=-w+\mathbf{S}^{1} w
\end{array}\right.
$$

Here the lower sign is chosen since the lower half-plane is associated with $\Omega^{-}$. The sum of equations in (16) results in $\mathbf{S}^{2} X=-2(1+\kappa)^{-1} w$, which is the following integral equation for the determination of unknown tractions under the stump

$$
\frac{1}{\pi i} \int_{-1}^{1} \frac{X(t)}{t-x} d t=-\frac{2}{1+\kappa} w(x), \quad 1<x<1+L
$$

After differentiation of (17) with respect to the contour variable one obtains the relationships between normal stresses and derivatives of displacements that are often used in contact problems for half-plane, e.g. [2].

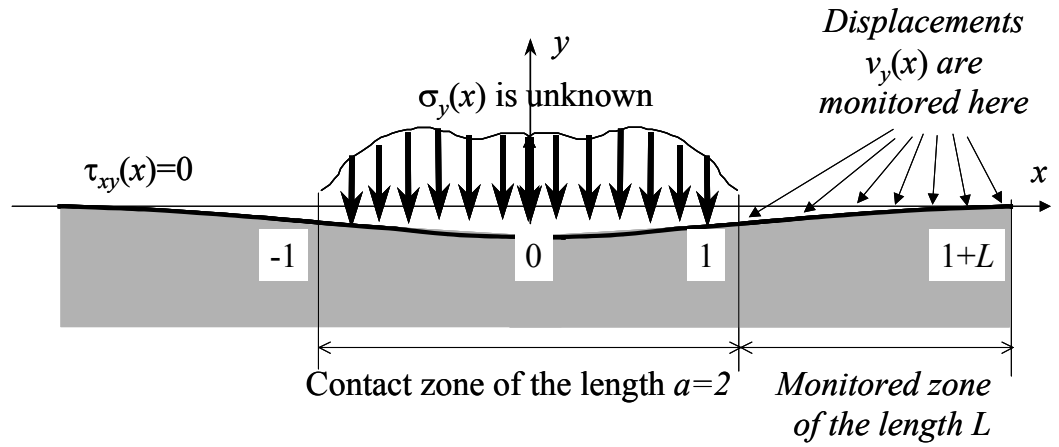

Figure 2: Scheme of displacements monitoring.

It should be noted that it is possible to derive an analytical solution of (17) if $L=\infty$, see [5], however no analytical solutions for the case of finite $L$ are known. We further apply a numerical approach similar to [12], which seems also to be suitable for more complex geometries.

Firstly one can rewrite (17) for the case of symmetrical polynomial load and for simplicity to neglect shear stresses as shown in Figure 2. This leads to the following integral equation

$$
\int_{0}^{1} \frac{N(t)}{t^{2}-x^{2}} d t=g(x), \quad 1<x<1+L, \quad g(x)=\frac{2 G}{1+\kappa} \frac{\pi}{x} u_{2}(x)
$$

Here $N(t)$ stands for the normal component of the resultant vector acting under the stump, the right hand side $g(x)$ depends on normal displacement only due to the absence of shear stresses. 
Equation (18) is further reduced by the collocation method to a system of linear algebraic equations $\mathbf{A C}=\mathbf{G}$ which solution (it depends on $L$ ) is found by the SVD method as follows

$$
\mathbf{C}=\mathbf{V}^{T} \mathbf{D}^{\prime \prime} \mathbf{U G}, \quad \mathbf{C}=\mathbf{C}(L)
$$

Here $\mathbf{U}(m \times n)$ and $\mathbf{V}(n \times n)$ are orthogonal matrices in the SDV decomposition $\mathbf{A}=\mathbf{U D V}^{\mathrm{T}}$ and $\mathbf{D}(n \times n)$ is a diagonal matrix formed by the singular values, $d_{j}$, placed in descending order, $d_{1} \geq d_{2} \geq \ldots \geq d_{n}$. the matrix $\mathbf{D}^{\prime}$ is the regularised inverse of $\mathbf{D}$ that has the rank $k$ : $\mathbf{D}^{\prime}=\operatorname{diag}\left\{d_{1}{ }^{-1}, d_{2}{ }^{-1}, \ldots d_{k}{ }^{-1}, 0 \ldots 0\right\}$.

\subsection{Statistical analysis}

This subsection presents the results of numerical experiments with synthetic data on displacement monitoring.

\subsubsection{Synthetic data}

To model displacement monitoring we introduce synthetic data as follows.

a) "True" stresses have been specified in the contact zone as polynomials of the fourth degree $N(x)=c_{0}+c_{1} x+c_{2} x^{2}+c_{3} x^{3}+c_{4} x^{4}$. In $j^{\text {th }}$ test all $c_{k}=0$ except $c_{j}=1$, $N^{\text {true }}(t)=(k+1) t^{k}$, which provides unit resultant force acting under the stump.

b) Ideal right hand sides corresponding to the loads above have been evaluated analytically on $(1,1+L)$ and than computed at collocation points (the number of which was set as $n=40$ for all $L$ varying within 0.01-10).

c) Distortion has been generated at each collocation point by introducing an independently generated random error normally distributed within $\pm 5 \%$.

Therefore in calculation the vector $\mathbf{G}$ had the components

$$
g_{m}=\sum_{k=0}^{4}(k+1) I_{k}\left(x_{m}, L\right)\left(1+\xi_{m}\right), \quad I_{k, m}(x, L)=\int_{0}^{1} \frac{t^{k} d t}{t^{2}-x^{2}}
$$

where $\xi_{m}, m=1, . ., n$ are Gaussian errors. The integrals $I_{k, m}$ have been evaluated exactly, which provides exact representation of the matrix $\mathbf{A}$, so the errors are only associated with the left hand side of the linear algebraic system of equations.

\subsubsection{Examples and statistics}

We study the following arrays of results in the set of 200 numerical experiments $(k=1 \ldots 200)$ for the resultant force

$$
P_{k}=\int_{0}^{1} N^{\text {recovered in } k \text {-test }}(x) d x, \quad \mathbf{P}=\left\{P_{k}\right\}
$$


and the divergence of the recovered and true contact stresses

$$
\delta_{k}=\left[\int_{0}^{1}\left(N^{\text {recovered in } k \text {-test }}(x)-N^{\text {true }}(x)\right)^{2} d x\right]^{\frac{1}{2}}, \quad \Delta=\left\{\delta_{k}\right\}
$$

Examples of contact stress recovery are presented in Figure 3 for the following cases

(a) - constant load with maximum and minimum errors of $E r r_{\max }=0.039, E r r_{\min }=-$ 0.041 normally distributed with Mean $=1.2 \cdot 10^{-3}, \underline{S t D e v}=0.03$, recovered resultant force is $P_{\text {rec }}=0.999$;

(b) - linear load with $E r r_{\max }=0.039, E r r_{\min }=-0.041$ Mean $=1.9 \cdot 10^{-3}$, StDev=0.07; $P_{\text {rec }}=1.002$;

(c) - parabolic load with $E r r_{\max }=0.047, E r r_{\min }=-0.03$ Mean=-6.0.10 $0^{-3}$, $\mathrm{StDev}=0.06 ; P_{\text {rec }}=1.006$;

(d) - cubic load with $E r r_{\max }=0.038, E r r_{\min }=-0.028 \mathrm{Mean}=4.8 \cdot 10^{-3}, \mathrm{StDev}=0.086$; $P_{\text {rec }}=1.005$

In all these examples the length of the monitored zones was $L=1$.

Statistical properties of (21) and (22) are presented in Figure 4 as functions of $L$.
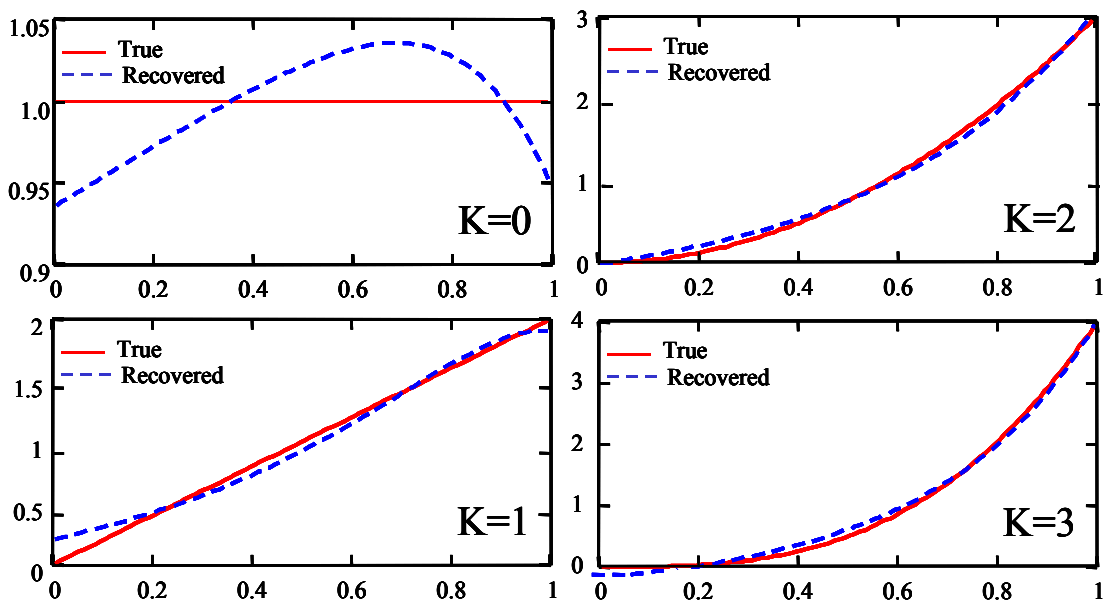

Figure 3: Examples of contact stresses recovering.

\section{Conclusions}

Solution of non-classical contact problem (5) for plane bodies is presented in operator form (14). The results of numerical experiments on the reconstruction of contact stresses demonstrate the SVD regularisation is an effective tool that provides stable numerical solutions. Statistical analysis of the results has been 
performed for the case of half-plane loaded by polynomial loads. It shows that resultant forces and traction distributions are better reconstructed for longer monitoring zones (Figure 4).

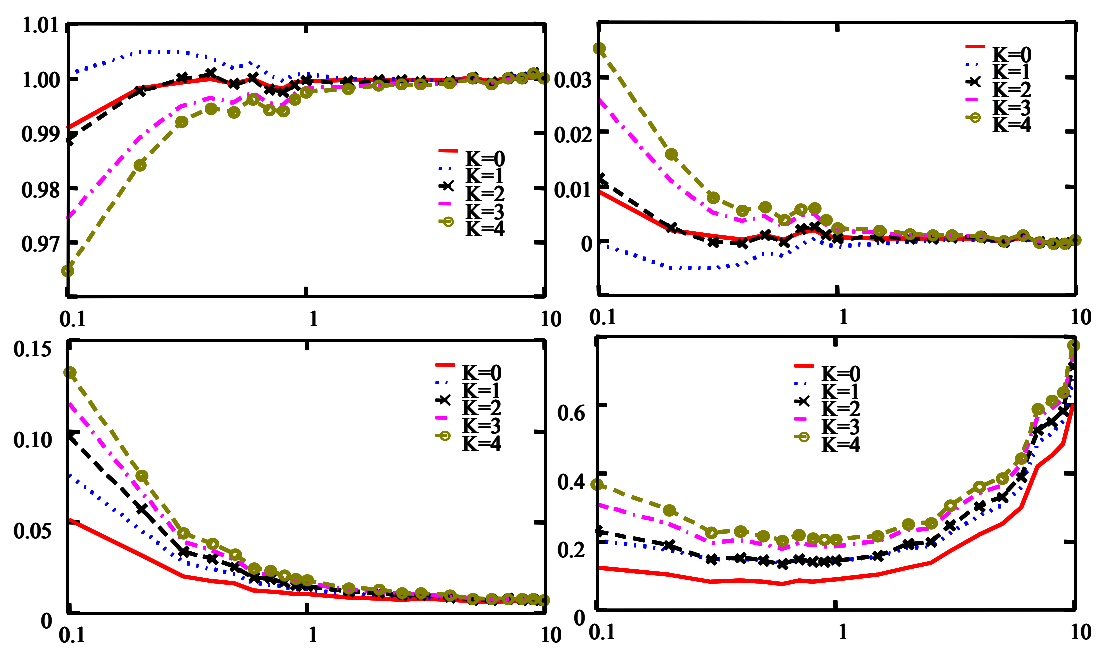

Figure 4: Means and standard deviations: $\mathbf{P}$ (left) and $\Delta$ (right).

\section{References}

[1] Muskhelishvili, N.I. Some basic problems of the mathematical theory of elasticity, P. Noordhoff, Groningen: the Netherlands, 1963.

[2] Johnson, K.L. Contact mechanics. Cambridge University Press, 1985.

[3] Shvab, A.A. 1989. Incorrectly posed static problems of elasticity, Mechanics of Solids. 24 (6), 98-106.

[4] Schwab, A. A. 1994. The inverse problem of elasticity theory: Application of the boundary integral equation for the holomorphic vector. Physics Of The Solid Earth. 30 (4), 342-348.

[5] Galybin, A.N., 1999. A non-classical plane elastic boundary value problem. Moving Boundaries V. Computational Modelling of Free and Moving Boundary Problems (Eds Sarler et al). WIT Press, Southampton, UK, 59-68.

[6] Tsvelodub, I. Yu. 2000. An inverse problem for an elastic] medium containing a physically non-linear inclusion. J. Appl. Maths Mechs. 64 (3), 407-412.

[7] Marin, L., L. Elliott, D.B. Ingham and D. Lesnic, 2001. Boundary element method for the Cauchy problem in linear elasticity, Engineering Analysis with Boundary Elements. 25 (9), 783-793. 
[8] Marin, L., D.N. Hào and D. Lesnic. 2002. Conjugate gradient-boundary element method for the Cauchy problem in elasticity. Quarterly Journal of Mechanics and Applied Mathematics. 55 (2), 227-247.

[9] Marin, L. and D. Lesnic. 2002. Regularized boundary element solution for an inverse boundary value problem in linear elasticity. Communications in Numerical Methods in Engineering. 18 (11), 817-825.

[10] Marin, L. and D. Lesnic. 2002. Boundary element solution for the Cauchy problem in linear elasticity using singular value decomposition. Computer Methods in Applied Mechanics and Engineering. 191 (6),3257-3270.

[11] Galybin, A.N., 2001. A Method for determination of stress distributions in the process zone ahead of a 2D crack. "Moving Boundaries VI" Computational Modelling of Free and Moving Boundary Problems (Eds B. Sarler and C. A. Brebbia). WIT Press, Southampton, UK 243-252.

[12] Galybin, A.N., 2002. Determination of softening law by measuring crack opening displacements. Structural Integrity and Fracture (Eds A.V. Dyskin et al). Swets \& Zeitlinger B.V., Lisse, The Netherlands, 35-41.

[13] Tikhonov, A.N. and Arsenin, V.Y. Solution of Ill-Posed Problems, New York: Winston, Wiley, 1977. 